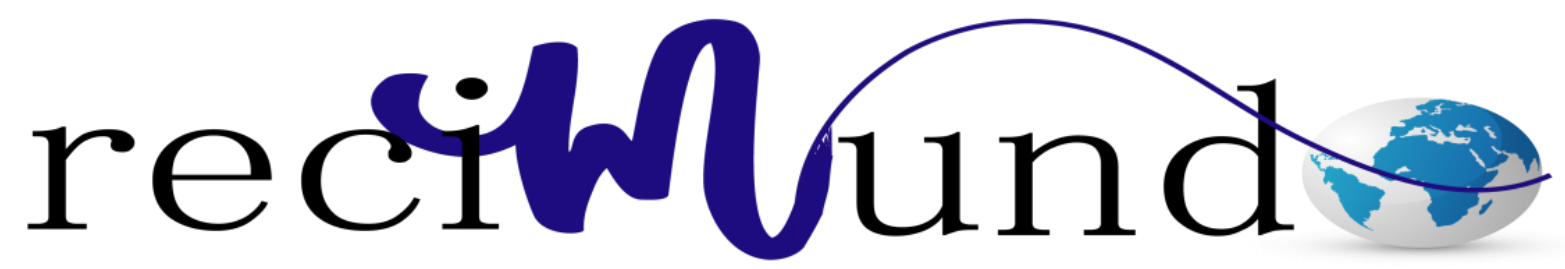

Revista Cientifica Mundo de la Investigación y el Conocimiento

Evelin Viviana Estacio Almeida ${ }^{\text {a }}$ K Katherine Jazmina Zambrano Zambrano ${ }^{\text {b; }}$

Katherine Gisella Bravo Bravo ${ }^{\text {c; }}$ Alexandra Elizabeth Rosales Cevallos ${ }^{\mathrm{d}}$

Bioética y aspectos médico-legales en la Unidad de Cuidados Intensivos

Bioethics and medical-legal aspects in the Intensive Care Unit

Revista Científica Mundo de la Investigación y el Conocimiento. Vol. 3 núm.3, septiembre, ISSN: 2588-073X, 2019, pp. 952-969

DOI: $10.26820 /$ recimundo/3.(3).septiembre.2019.952-969

URL: http://recimundo.com/index.php/es/article/view/556

Código UNESCO: 7102 Ética de Individuos

Tipo de Investigación: Artículo de Revisión

Editorial Saberes del Conocimiento

Recibido: $15 / 05 / 2019$

Aceptado: 23/06/2019

Publicado: 30/09/2019

Correspondencia: nileve2611@ hotmail.com

a. Médico; Investigador Independiente; Guayaquil, Ecuador; nileve2611@ hotmail.com

b. Médica Cirujana; Investigador Independiente; Guayaquil, Ecuador; katyjas_1711@ hotmail.com

c. Médico Cirujano; Investigador Independiente; Guayaquil, Ecuador; katherine bravob@ hotmail.com

d. Médico; Investigador Independiente; Guayaquil, Ecuador; eli-ta_200@ hotmail.com 


\section{Bioética y aspectos médico-legales en la Unidad de Cuidados Intensivos}

Vol. 3, núm. 3., (2019)

Evelin Viviana Estacio Almeida; Katherine Jazmina Zambrano Zambrano; Katherine Gisella Bravo Bravo; Alexandra Elizabeth Rosales Cevallos

\section{RESUMEN}

El desarrollo de la tecnología en la medicina permite lograr un soporte de vida, sin tomar en cuenta la calidad de la misma y la decisión del paciente o enfermo. Se desarrolló la bioética, primero para la investigación y luego para proteger al individuo en la práctica clínica; en UCI el paciente debe ser manejado con el criterio del intensivista y su equipo en consonancia con el respeto a lo que quiere o necesita el enfermo para su egreso o el bien morir. El personal de salud debe explicar que está pasando, probabilidades, procedimientos diagnósticos y terapéuticos para luego tomar las decisiones en consenso con el enfermo, sí es posible y su familia, quienes a veces desconocen los deseos del paciente. Debe respetarse la autonomía, consentimiento informado, beneficencia, calidad de vida. Acudir al Comité de Bioética cuando sea necesario y para todo lo anterior, es necesario que el profesional de salud conozca y maneje la bioética, manteniendo la educación continua sobre el tema, en pregrado, postgrado y práctica diaria. En nuestros países hay desconocimiento del tema y poco interés en aprender sobre el mismo.

Palabras claves: Autonomía; Bioética; Beneficencia; Consentimiento; Calidad de vida; Limitación de esfuerzo terapéutico. 


\title{
Bioética y aspectos médico-legales en la Unidad de Cuidados Intensivos
}

Vol. 3, núm. 3., (2019)

Evelin Viviana Estacio Almeida; Katherine Jazmina Zambrano Zambrano; Katherine Gisella Bravo Bravo; Alexandra Elizabeth Rosales Cevallos

\begin{abstract}
The development of technology in medicine allows to achieve a life support, without taking into account the quality of it and the decision of the patient or patient. Bioethics was developed, first for research and then to protect the individual in clinical practice; In the ICU, the patient must be managed according to the criteria of the intensivist and his team in line with respect for what the patient wants or needs for his discharge or for the good to die. The health staff must explain what is happening, probabilities, diagnostic and therapeutic procedures and then make decisions in consensus with the patient, if possible and his family, who sometimes do not know the patient's wishes. Autonomy, informed consent, beneficence, quality of life must be respected. Go to the Bioethics Committee when necessary and for all the above, it is necessary for the health professional to know and manage bioethics, maintaining continuous education on the subject, in undergraduate, postgraduate and daily practice. In our countries there is ignorance of the subject and little interest in learning about it.
\end{abstract}

Key words: Autonomy; Bioethics; Beneficence; Consent; Quality of life; Limitation of therapeutic effort. 


\section{Bioética y aspectos médico-legales en la Unidad de Cuidados Intensivos}

Vol. 3, núm. 3., (2019)

Evelin Viviana Estacio Almeida; Katherine Jazmina Zambrano Zambrano; Katherine Gisella Bravo Bravo; Alexandra Elizabeth Rosales Cevallos

\section{Introducción.}

El gran desarrollo de la medicina desde el punto de vista tecnológico que permite prolongar la vida con soporte avanzado, sin tomar en cuenta la calidad de vida, hace de la bioética algo fundamental para proteger al enfermo.

Una de las áreas más difíciles de manejar es la Unidad de Cuidados Intensivos (UCI); el diagnóstico, estado de conciencia y pronóstico del paciente en conjunto con su entorno familiar, hacen que los criterios de ingreso, egreso, conducta terapéutica y procedimientos diagnósticos sean decisiones que deben ser bien explicadas y discutidas con el paciente (no siempre es posible, por su estado de conciencia) o su familia.

El avance científico no puede estar sobre la parte humana de la práctica médica, el médico debe estar en sintonía con los deseos del paciente y la calidad de vida que merece todo ser humano. Los médicos tienen como objetivo salvar vidas, pero deben aprender que a veces no es posible y ahí es cuando la bioética se vuelve parte de esa decisión para brindar un fallecimiento digno.

\section{Unidad de Cuidados Intensivos}

Estas unidades tienen una historia que se remonta a 1854, con la enfermera Florence Nigthingale quien separó a los soldados más graves de aquellos con heridas leves, disminuyendo la mortalidad. En los años 50, Dinamarca, Suecia y Francia desarrollaron los primeros equipos de ventilación mecánica debido a la epidemia de poliomelitis. El Dr. Peter Safar, considerado el primer intensivista, creó las primeras unidades de cuidado intensivo, en Baltimore en 1958 y Pittsburg en 1961. (Aguilar Garcia \& Martínez T., 2017) En las décadas siguientes se desarrollaron 


\section{Bioética y aspectos médico-legales en la Unidad de Cuidados Intensivos}

Vol. 3, núm. 3., (2019)

Evelin Viviana Estacio Almeida; Katherine Jazmina Zambrano Zambrano; Katherine Gisella Bravo Bravo; Alexandra Elizabeth Rosales Cevallos

unidades en todo el mundo, primero en los países desarrollados, con unidades para recién nacidos y niños, quemados, postoperatorios de neurocirugía y cirugía cardiaca y luego ya fueron las unidades de cuidados intensivos. (Angueyra Díaz, 2013)

La UCI se puede definir como una organización multidisciplinaria, que se ubica en un espacio determinado del hospital para brindar condiciones de seguridad, calidad y eficiencia a pacientes que requieran soporte ventilatorio y con falla de al menos dos órganos y son susceptibles de recuperación. (Sanidad, 2010)

\section{Medicina Crítica o Intensiva}

Esta especialidad es la encargada de los pacientes en riesgo de perder la vida, aliviar el sufrimiento, evitar daños, con el objetivo que el paciente sobreviva como consecuencia de utilizar el apoyo de soporte vital y los cuidados intensivos. (Caballero Velarde, 2012) (Bembibre Taboada, 2003)

El paciente para UCI es aquel que está en riesgo de perder la vida o en estado crítico; puede estar inestable, con enfermedad grave, descompensación de una enfermedad crónica, con posibilidad de revertir la enfermedad o condición y requieren asistencia continua de enfermería, equipos de soporte con vigilancia las 24 horas. (Aguilar Garcia \& Martínez T., 2017)

Sin olvidar que el paciente de UCI también puede fallecer y parte de la bioética es como el personal de la unidad maneje la situación con el paciente y la familia. (Sandoval-Gutiérrez, 2017) 


\section{Bioética y aspectos médico-legales en la Unidad de Cuidados Intensivos}

Vol. 3, núm. 3., (2019)

Evelin Viviana Estacio Almeida; Katherine Jazmina Zambrano Zambrano; Katherine Gisella Bravo Bravo; Alexandra Elizabeth Rosales Cevallos

Antecedentes de la Bioética

En 1914, una sentencia sobre una demanda contra el Hospital de New York, concluyo que un adulto con mente sana tiene derecho a determinar lo que hará con su cuerpo. (Luce \& White, 2009)

El Código de Núremberg en 1947, sobre la investigación con humanos, crea la obligación del consentimiento voluntario. (Littewka, 2006)

Otro proceso judicial, en California, 1957, determina que se debe informar a los pacientes para que decidan libremente sobre los procedimientos médicos a los que se someterán. (Angueyra Díaz, 2013)

El Informe Belmont, 1978, considera que para la investigación científica debe existir respeto, beneficencia y justicia hacia las personas; además, la utilidad del consentimiento informado, evaluar riesgo-beneficio y selección imparcial del sujeto a investigar. Luego en 1979, se publica el libro Principios de la Ética Biomédica que propone 4 principios: respeto a la autonomía, no hacer daño, justicia, beneficio. (Angueyra Díaz, 2013)

\section{¿Qué es Bioética?}

Según la Organización Mundial de la Salud y OPS la Bioética es una disciplina que trata sobre los problemas éticos en la salud, desde investigación en seres humanos, diseño e implementación de políticas de salud y brindar atención médica. Es un análisis de principios y 


\section{Bioética y aspectos médico-legales en la Unidad de Cuidados Intensivos}

Vol. 3, núm. 3., (2019)

Evelin Viviana Estacio Almeida; Katherine Jazmina Zambrano Zambrano; Katherine Gisella Bravo Bravo; Alexandra Elizabeth Rosales Cevallos

criterios éticos, usada como guía en las distintas áreas prácticas de la salud. La OPS tiene un Programa Regional de Bioética establecido desde 1994. (Organización Panamericana de la Salud)

Otra definición nos dice que es una "Disciplina científica que estudia los aspectos éticos de la medicina y la biología en general, así como las relaciones del hombre con los restantes seres vivos". (Van Rensselaer, 1971)

Según la RAE es el "estudio de los problemas éticos originados por la investigación biológica y sus aplicaciones".

Tenemos la definición de ética médica que según el Dr. Ruy Pérez es “el conjunto de valores, principios morales y de acciones relevantes del personal responsable de la salud (médicos, enfermeras, técnicos y funcionarios) dirigidos a cumplir con los objetivos de la medicina". (Vásquez Ortiz, Esparza, Duffo Villegas, \& Ibarra Zazueta, 2005)

\section{Otras definiciones importantes en Bioética}

Historia Clínica: es un conjunto de documentos con los datos, valoraciones e informaciones sobre la situación y evolución clínica de un paciente ingresado en un centro asistencial. Debe tener la identificación del personal que atienda al paciente, estar bien custodiada y disponible para cuando el paciente, familia o representante legal solicite información.

Consentimiento informado: forma parte de la historia clínica, en el cual el paciente o su representante legal mediante su firma acepta o niega al procedimiento diagnóstico o terapéutico luego que el médico le explicó ventajas y desventajas del mismo. 


\section{Bioética y aspectos médico-legales en la Unidad de Cuidados Intensivos}

Vol. 3, núm. 3., (2019)

Evelin Viviana Estacio Almeida; Katherine Jazmina Zambrano Zambrano; Katherine Gisella Bravo Bravo; Alexandra Elizabeth Rosales Cevallos

El consentimiento está formado por: 1. Identificación del enfermo y médico que indica y pide consentimiento. 2. Nombre, identificación, objetivo del procedimiento diagnóstico o terapéutico, riesgos, beneficios y alternativas. 3. Informar el derecho a aceptar o rechazar el procedimiento o poner límites. 4. Confidencialidad. 5. Fecha del consentimiento. 6. Apartado para consentimiento de representante legal. 7. Documento debe ser por duplicado. También, se firma un consentimiento aparte para recibir anestesia. (Sanidad, 2010) (Chancay Aragundi \& Escuntar Villacís, 2015)

Autonomía: el paciente entiende su condición y decide su tratamiento sin recibir coacciones. (Hernández Rastrolloa, 2008)

Beneficencia: hacer el bien, por parte del médico, respetando la autonomía del paciente. (Hernández Rastrolloa, 2008)

Calidad de vida: es la percepción del paciente de vivir en condiciones dignas, según su criterio. (Hernández Rastrolloa, 2008)

Comité de bioética asistencial: equipo que permite resolver conflictos dentro de la práctica clínica. Tienen como función proteger los derechos de los pacientes, facilitar el proceso cuando haya conflictos éticos, elaborar recomendaciones para situaciones habituales, promover educación en bioética. (Hernández Rastrolloa, 2008)

Deontología: conjunto de principios y reglas éticas que deben regir la conducta profesional del médico. 


\section{Bioética y aspectos médico-legales en la Unidad de Cuidados Intensivos}

Vol. 3, núm. 3., (2019)

Evelin Viviana Estacio Almeida; Katherine Jazmina Zambrano Zambrano; Katherine Gisella Bravo Bravo; Alexandra Elizabeth Rosales Cevallos

Distanasia: prologar agonía, utilizando de manera abusiva los equipos de tecnología avanzada.

Limitación del esfuerzo terapéutico (LET): decisión de retirar o no iniciar soporte vital en paciente con mal pronóstico. Se brindan solo cuidados paliativos.

\section{Importancia}

Con respeto al área de UCI, tomar las decisiones diagnósticas y/o terapéuticas es más complicado, ya que el paciente puede no ser capaz de expresar sus deseos y es el médico o la familia quienes deciden, a veces sin realmente conocer que desearía esa persona o sin tomarlo en cuenta.

La bioética es necesaria, porque el médico se fue alejando de la parte humana y se afianzó en la tecnología; transformó al paciente en objeto de estudio y olvidó que es una persona. El enfermo es una persona con derecho a expresar sus deseos de conocer, entender su situación y ser capaz de tomar la decisión sobre las alternativas diagnosticas y/o terapéuticas, acorde a sus creencias.

En Estados Unidos, para el mejor manejo del paciente crítico, se establecen criterios: subjetivo, de juicio sustitutivo y el de mayor beneficio. El criterio subjetivo es más difícil, porque se basa en lo que haya expresado previamente el paciente de manera verbal o por escrito para determinada situación y hay que interpretarla. El de juicio sustitutivo, el representante toma la decisión, depende de sí el paciente alguna vez expresó su opinión a las situaciones similares y de la honestidad de ese representante. Y, por último, el criterio del mayor beneficio que implica dar 


\section{Bioética y aspectos médico-legales en la Unidad de Cuidados Intensivos}

Vol. 3, núm. 3., (2019)

Evelin Viviana Estacio Almeida; Katherine Jazmina Zambrano Zambrano; Katherine Gisella Bravo Bravo; Alexandra Elizabeth Rosales Cevallos

bienestar al paciente, por un lado, aliviando el sufrimiento y/o recuperar la calidad de vida. (Kuczewski, 2004) (Arnold \& Kellum, 2003) (Hernández-Tejedor, 2014)

Por ejemplo, en España la familia busca proteger al paciente, escondiendo el diagnóstico o gravedad, teniendo la decisión familiar el mismo valor que el consentimiento informado, pudiendo haber diferencia entre lo que quisiera el paciente y lo que pide la familia. Además, la ley permite tomar decisiones en contra del deseo de la familia, sí se considera que no actúan a favor del paciente. (Hernández-Tejedor, 2014)

El paciente y/o familia deben ser informados sobre que es la UCI, los cuidados que recibe el paciente en esa área, firmar el consentimiento informado. Todo debe ser explicado en un lenguaje sencillo y libre de tecnicismos. Debe haber una persona responsable de brindar la información pertinente y evolución diaria. (Sanidad, 2010)

El hospital y el Comité de Bioética deben tener políticas sobre la asistencia de los pacientes según su religión o creencias se nieguen a recibir algún tratamiento, adaptando el consentimiento. (Sanidad, 2010)

Todo centro de salud debe tener un comité de bioética para resolver las dudas que se presenten en el manejo del paciente intrahospitalario.

En América Latina, la relación médico paciente tiene un carácter paternalista, por lo cual hay un retraso en el manejo de la bioética. (Chancay Aragundi \& Escuntar Villacís, 2015) 


\section{Bioética y aspectos médico-legales en la Unidad de Cuidados Intensivos}

Vol. 3, núm. 3., (2019)

Evelin Viviana Estacio Almeida; Katherine Jazmina Zambrano Zambrano; Katherine Gisella Bravo Bravo; Alexandra Elizabeth Rosales Cevallos

En resumen,

- Los derechos de los pacientes críticos son: Recibir atención especializada para minimizar el riesgo de morir o de incapacidad, Recibir la información correcta de su condición, Rechazar tratamientos dentro del marco legal, Recibir asistencia religiosa según su credo, Confidencialidad sobre su enfermedad, Recibir atención personalizada, respetuosa de médicos y enfermeras. (Bembibre Taboada, 2003)

- Las decisiones bioéticas deben tomar en cuenta riesgo vs daño y beneficio, optimizar el diagnóstico, estado de competencia del paciente, informar a la familia, determinar calidad de vida acorde al paciente, manejar un criterio único dentro del equipo, uso razonable de los recursos, permitir a la familia adaptarse cuando ya no haya nada que hacer, cumplir correctamente con los cuidados paliativos, no abandonar. (Caballero Velarde, 2012)

\section{Metodología}

Revisión bibliográfica de artículos originales, revisiones, trabajos de grado, sitios web considerados válidos u oficiales para la toma de datos e información.

\section{Resultados}

Bioética y Aspectos Medico Legales en UCI en América Latina

En México, se ha visto que el ingreso de un paciente a UCI puede ser por razones distintas a los criterios clínicos o médicos; por lo cual se pierde el manejo correcto del paciente. Se incrementan costos y ocupando una cama al enfermo que sí lo requiere. El costo día de un paciente 


\section{Bioética y aspectos médico-legales en la Unidad de Cuidados Intensivos}

Vol. 3, núm. 3., (2019)

Evelin Viviana Estacio Almeida; Katherine Jazmina Zambrano Zambrano; Katherine Gisella Bravo Bravo; Alexandra Elizabeth Rosales Cevallos

en UCI es 34.509 pesos mexicanos sin tomar en cuenta costos secundarios por co-morbilidades nosocomiales o propias del paciente. (Aguilar Garcia \& Martínez T., 2017)

Para 1991, en el Código de Ética Médica de Colombia, se estableció que en pacientes críticos: se solicitara consentimiento para los procedimientos, no se realizarán sin justificación y se explicará anticipadamente las consecuencias al paciente o a su representante. En 1994, en Colombia se sentenció que el Consentimiento Informado es un elemento que permite al paciente manifestar sus derechos y hacerlo participe de las decisiones relativas a su salud bajo la guía de su médico. (Angueyra Díaz, 2013)

Un trabajo realizado en 13 hospitales (públicos y privados) de Bogotá encuestando a médicos y enfermeras, sobre bioética, concluyó: 40\% de personal de UCI no recibió la asignatura bioética, $19,1 \%$ dice que no hay comité de ética en su centro, $16 \%$ dice que no sabe, $42 \%$ de conflictos éticos en UCI, 29,5\% no consulta a la familia para la toma de decisiones; por tanto, el personal de salud debe recibir educación sobre bioética para mejorar la atención médica. (Rincón, 2008)

En Cuba, el manejo del paciente crítico es gratuito, sin escatimar recursos, utilizando la tecnología de punta disponible. (Bembibre Taboada, 2003)

Un trabajo realizado en personal de UCI, en hospitales de Cuba ofreció los siguientes resultados sobre bioética: 100\% no maleficencia, 97,6\% autonomía, 95,2\% beneficencia, 92,8\% justicia. Todos los encuestados recibieron la asignatura de bioética y conocen el concepto de bioética. (Alonso, Alonso, \& Elvis, 2014) 


\section{Bioética y aspectos médico-legales en la Unidad de Cuidados Intensivos}

Vol. 3, núm. 3., (2019)

Evelin Viviana Estacio Almeida; Katherine Jazmina Zambrano Zambrano; Katherine Gisella Bravo Bravo; Alexandra Elizabeth Rosales Cevallos

\section{Ecuador}

En 2007 la OPS y OMS otorgaron a Ecuador, apoyo técnico y financiero para diagnosticar la situación de la bioética en pre y postgrado de todas las universidades. Ya para 2009, existía la cátedra de bioética en el tercer y cuarto nivel de formación profesional. (Cardenas, 2009)

Desde 2013 existe la Comisión Nacional de Bioética en Salud de Ecuador. Plantean que la bioética se debe utilizar para "definir lo correcto o incorrecto en las ciencias biológicas". Su trabajo es crear la bioética de la salud para mantener la dignidad, derechos, seguridad y bienestar de las personas en las aras de salud pública, investigación y práctica asistencial. (Arturo, Cárdenas, Cifuentes, \& Duque, 2017)

Dentro del manejo del paciente crítico, esta Comisión plantea el manejo correcto de la muerte en UCI; el médico debe ser el modelo de respeto a vida y muerte digna, debe brindar atención humanizada, ofrecer información oportuna a los familiares, sin paternalismo y prepotencia, preparar el ambiente, respetar los rituales religiosos, suspender procedimientos no necesarios. Con respecto al consentimiento, la evolución ha sido lenta. Apenas en 2008, es cuando aparece una referencia en la Constitución Política. (Arturo, Cárdenas, Cifuentes, \& Duque, 2017)

Se realizó un trabajo de investigación para determinar el conocimiento sobre bioética en enfermeras del área de emergencias. Sus resultados determinaron que había un bajo nivel de conocimientos sobre el tema, no lo aplicaban al manejo de los pacientes y tenían poco interés de participar en el Comité de Bioética de la institución. (Chancay Aragundi \& Escuntar Villacís, 2015) 


\section{Bioética y aspectos médico-legales en la Unidad de Cuidados Intensivos}

Vol. 3, núm. 3., (2019)

Evelin Viviana Estacio Almeida; Katherine Jazmina Zambrano Zambrano; Katherine Gisella Bravo Bravo; Alexandra Elizabeth Rosales Cevallos

América Latina tiene una práctica médica paternalista, donde el criterio del médico prevalece. Estudios realizados en Argentina y Brasil sobre manejo de paciente crítico pediátrico, reportan que solo el 9\% de las familias participa en la toma de decisiones. (Morales, 2015)

Un trabajo de grado con personal de UCI, presentó en sus resultados:

La decisión de LET se tomaba con criterios de probabilidad no dé certeza; sin existir marco legal nacional o institucional con poca participación del comité de bioética.

El personal de UCI entrevistado, manifestaba poco interés en actualización sobre la bioética con respecto a la humanización del manejo del paciente.

La atención brindada es principalmente técnica, no se integra enfermedad y ser humano. (Bonilla Merizalde, 2010)

\section{Conclusiones}

La bioética es fundamental en la práctica médica:

$\checkmark$ Por un lado, el avance tecnológico alejó al médico del paciente desde el punto de vista humano y lo convirtió en objeto de estudio o problema a resolver.

$\checkmark$ Por otro lado, le permite al paciente estar informado y poder tomar decisiones sobre su salud.

La condición del paciente de UCI amerita poner en práctica todos los componentes de la bioética, por su enfermedad y pronóstico, por el estado de conciencia y el entorno familiar. 


\section{Bioética y aspectos médico-legales en la Unidad de Cuidados Intensivos}

Vol. 3, núm. 3., (2019)

Evelin Viviana Estacio Almeida; Katherine Jazmina Zambrano Zambrano; Katherine Gisella Bravo Bravo; Alexandra Elizabeth Rosales Cevallos

La medicina latina tiene un fuerte componente paternalista.

Hay desconocimiento sobre bioética dentro del personal de salud, hasta desconocen sobre la existencia del Comité de Bioética.

Según los trabajos revisados, en Ecuador hay desconocimiento y poco interés en la bioética.

\section{Recomendaciones}

Incentivar la asignatura bioética en las universidades, tanto en el área de pregrado como postgrado.

Debe haber actualizaciones sobre bioética durante el ejercicio profesional del personal de salud, especialmente en quienes manejan UCI y emergencias.

El Comité de Bioética debe ser conocido y utilizado para resolver o prevenir complicaciones éticas y/o legales.

Las personas deben expresar sus deseos, en las enfermedades crónicas, terminales, para evitar conflictos o no cumplir sus deseos en caso de perder la conciencia.

El consentimiento informado debe ser bien realizado, por lo cual se requiere educar al personal de salud (el médico tratante debe ser quien se encargue).

El ingreso de paciente a UCI y su manejo debe ser responsabilidad del equipo y el médico intensivista el líder. 


\section{Bioética y aspectos médico-legales en la Unidad de Cuidados Intensivos}

Vol. 3, núm. 3., (2019)

Evelin Viviana Estacio Almeida; Katherine Jazmina Zambrano Zambrano; Katherine Gisella Bravo Bravo; Alexandra Elizabeth Rosales Cevallos

La familia y paciente deben estar bien informados sobre la enfermedad, procedimientos diagnósticos y terapéuticos con sus consecuencias.

En lo posible, familia, paciente y medico deben discutir qué es lo mejor para el enfermo.

Los avances tecnológicos, equipos y pruebas diagnósticas deben utilizarse con criterio.

El médico debe considerar calidad de vida del enfermo al egreso, para decidir conductas dentro de UCI.

El personal de UCI debe recibir apoyo y entrenamiento para el manejo del stress laboral como el manejo al paciente y su familia.

\section{Bibliografía.}

Aguilar Garcia, C., \& Martínez T., C. (2017). La realidad de la Unidad de Cuidados Intensivos. Medicina Critica, 31(3), 171-173.

Alonso, A. L., Alonso, O., \& Elvis, L. (2014). Dilemas éticos de las decisiones médicas en cuidados intensivos. Acta Médica del Centro, 37-45.

Angueyra Díaz, H. (noviembre de 2013). Problemas bioéticos en las unidades de cuidados intensivos. Lima: Guzlop editores.

Arnold, R., \& Kellum, J. (2003). Moral justifications for surrogate decision making in the intensive care unit: Implications and limitations. Crit Care Med, S347-S353.

Arturo, L., Cárdenas, V., Cifuentes, A. C., \& Duque, L. (2017). Criterios Bioéticos. Comisión Nacional de Bioética en Salud Ecuador, 2017. Loja: Cátedra Unesco Ética y Sociedad en la Educación Superior, Universidad Técnica Particular de Loja.

Bembibre Taboada, R. (2003). Aspectos Éticos-Bioéticos en la Atención del Paciente Crítico. Revista Cubana de Medicina, 5-11. 


\section{Bioética y aspectos médico-legales en la Unidad de Cuidados Intensivos}

Vol. 3, núm. 3., (2019)

Evelin Viviana Estacio Almeida; Katherine Jazmina Zambrano Zambrano; Katherine Gisella Bravo Bravo; Alexandra Elizabeth Rosales Cevallos

Bonilla Merizalde, J. (2010). Investigación realizada en las Unidades de Cuidados Intensivos de los Hospitales Eugenio Espejo y Carlos Andrade Marín. Quito Enero 2010 - Julio 2011. Quito : Universidad Libre Internacional de las Américas.

Caballero Velarde, M. (2012). Estrategia ante el reto bioético del paciente crítico. Cirujano General, S97-S99.

Cardenas, S. (2009). “La bioética en la formación de recursos humanos de la salud”. Situación actual y desafíos en el Ecuador. Quito: Comisión Nacional de Bioética.

Chancay Aragundi, M., \& Escuntar Villacís, A. (2015). Conocimientos de las enfermeras sobre bioética y su aplicación en el cuidado a pacientes de emergencia del Hospital General Enrique Garcés, enero-junio 2012. Quito : Universidad Central de Ecuador.

Hernández Rastrolloa, R. y. (2008). Glosario de términos y expresiones frecuentes de bioética en la práctica de cuidados intensivos pediátricos. Anales de Pediatria, 393-400.

Hernández-Tejedor, A. (2014). Revisión sobre bioética en la Unidad de Cuidados Intensivos: sobre la autonomía y el papel de los familiares y los representantes legales. Medicina Intensiva, 104-110.

Kuczewski, M. (2004). From informed consent to substituted judgment: Decision-making at the end-of-life. HEC Forum, 27-37.

Littewka, S. (2006). Planeación ética de los experimentos con seres humanos. Revista Colombiana d Bioética, 131-140.

Luce, J., \& White, D. (2009). Luce, John, White, DA History of Ethics and Law in the Intensive Care Unit. Crit Care Med 2009; 25: 221. Critical Care Medicine, 221.

Morales, G. (2015). Limitación del esfuerzo terapéutico en cuidados intensivos pediátricos. Revista Chilena de Pediatria, 56-60.

OPS. (2017). Programa Regional de Bioética. Washington: OMS. Obtenido de https://www.paho.org/hq/index.php?Itemid=4124\&lang=es

Real Academia de la Lengua Española. (15 de 09 de 2019). Obtenido de https://dle.rae.es

Rincón, M. y. (2008). Educación, Bioética y toma de decisiones éticas en unidades de cuidados intensivos. Revista Latinoamericana de Bioética, 114-123.

Sandoval-Gutiérrez, J. (2017). Muerte y bioética en la unidad de cuidados intensivos. Gaceta Médica de México, 520-521.

Sanidad, M. d. (2010). Unidad de Cuidados Intensivos. Estandares y Recomendaciones. Madrid: Ministerio de Sanidad y Politica Social. 


\section{Bioética y aspectos médico-legales en la Unidad de Cuidados Intensivos}

Vol. 3, núm. 3., (2019)

Evelin Viviana Estacio Almeida; Katherine Jazmina Zambrano Zambrano; Katherine Gisella Bravo Bravo; Alexandra Elizabeth Rosales Cevallos

Van Rensselaer, P. (1971). Diccionario de la Real Academia Española.

Vásquez Ortiz, E., Esparza, E., Duffo Villegas, M., \& Ibarra Zazueta, L. (2005). Ética médica y aspectos legales. Acta Ortopédica Mexicana, 132-134. 